\title{
LA CRITICITE
}

\section{PREVENTION, ACCIDENTS ET INCIDENTS, ALARME, DOSIMETRIE *}

\author{
par A. GEY ** \\ (Manuscrit ręu le 18 juin 1973 )
}

\section{Chers Collègues,}

C'est une tradition de la Société Française de Radioprotection que de donner la parole à votre Président-élu pour une allocution qui - entre autres buts a celui de meubler le temps mort nécessaire au dépouillement du vote. Succédant ainsi à neuf éminents prédécesseurs ***, je me suis trouvé embarrassé pour découvrir un sujet d'allocution qui n'ait point été déjà traité avec la maîtrise qu'on leur connaît par ces personnalités de renom. Me souvenant alors que j'ai eu l'honneur et le plaisir de siéger pendant dix années environ à la Sous-Commission des Masses Critiques, et malgré la proximité d'une réunion scientifique très complète et intéressante organisée, d'une part, par MM. DUHAMEL et JACQUEMIN, présidée, d'autre part, par M. LECORCHÉ, j'ai pensé qu'une synthèse sur le thème de la criticité serait peut-être profitable au moins à ceux qui n'ont eu ni le loisir, ni la chance d'assister à cette précédente réunion. C'est pourquoi je vous propose d'évoquer aujourd'hui l'essentiel de ce qu'est l'état critique, ce qui provoque son existence et ce qui s'y oppose, comment prévenir son apparition, quels accidents ou incidents ont mis en échec cette prévention, de quel système d'alarme on dispose et comment apprécier les dommages personnels éventuellement provoqués par une excursion critique accidentelle.

Et tout d'abord, qu'est-ce que l'état critique?

\section{DÉFINITION DE L'ÉTAT CRITIQUE}

L'état critique est l'état d'un milieu multiplicateur dans lequel le bilan instantané des apparitions et des disparitions de particules est nul; en l'occurrence le milieu multiplicateur est fissile et les objets multipliés sont les neutrons [I] (tableau I).

* Exposé de l'auteur à l'issue dẹ̦'l'assemblée générale ordinaire de la S.F.R.P., le I 2 juin 1973.

** C.E.A.-S.E.S.R. - Centre d'Etudes Nucléaires de Saclay, B.P. 2 - 9r Gif-sur-Yvette.

*** MM. Chassende-Baroz, Bonet-Maury, Jammet, Delpla, Blanc, Duhamel, Avargues, Candes, Marble.

RADIOPROTECTION, VOL. $8-\mathrm{N}^{\circ}{ }_{2}$ 


\section{TABLEAU 1}

$$
\left.d n=(K-1) n \cdot \frac{d t}{\theta}\right] \quad R e ́ f \cdot[1], p \cdot 40
$$

avec: ( $n=$ nombre total de neutrons présents à l'instant $t$

$(\theta)$ vie moyenne de ces neutrons

$(K=$ coefficient de multiplication

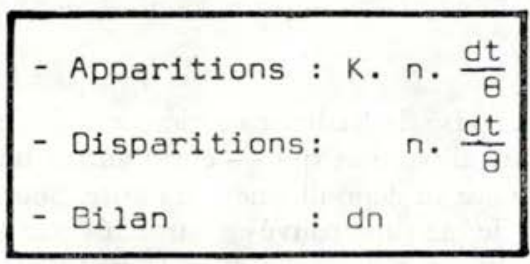

On remarquera en passant que s'il existait d'autres particules multipliables par des processus autres que la fission, la définition de l'état critique, dans le sens que nous lui accordons, resterait la même. On remarquera également que pour qu'il y ait multiplication, il faut au moins un évènement initiateur car le produit de zéro par n'importe quoi de fini donne toujours zéro : cette remarque n'est pas dénuée d'intérêt pratique car on peut dépasser l'état critique - donc rendre un milieu surcritique - sans déclencher de conséquences matérielles pourvu qu'il y ait rigoureusement absence d'initiation. Il n'est pas nécessaire de s'appesantir longtemps sur cette constatation pour imaginer l'emploi qu'on peut en faire dans les processus explosifs. C'est pourquoi, de façon un peu osée, on pourrait dire que l'état critique est un état « virtuel », que révèle l'injection d'un neutron initial efficace.

Toujours de façon un peu osée, l'état critique pourrait être qualifié d'“ idéal » en donnant à ce qualificatif le sens qu'on applique à la ligne droite, par définition sans épaisseur. En effet, si on photographiait en quelque sorte la situation neutronique d'un milieu critique initié, avec un temps de pose infiniment court, on aurait selon le moment choisi un tout petit peu plus ou un tout petit peu moins de neutrons que ne le voudrait la stricte application de la définition. Cela tient au fait que la durée de vie d'un neutron dans le milieu n'est pas nulle et que le trajet qui le conduit à la capture ou à l'évasion n'est pas identique selon la position qu'il occupe à sa naissance dans le milieu (fig. I).

Etat " virtuel », état " idéal », l'état critique répond à sa définition si on la corrige ainsi : c'est l'état d'un milieu multiplicateur dans lequel le bilan instantané des apparitions et des disparitions est, dans le temps, statistiquement nul.

Mais quelles sont ces apparitions, quelles sont ces disparitions? 


\section{dn (Bilan)}
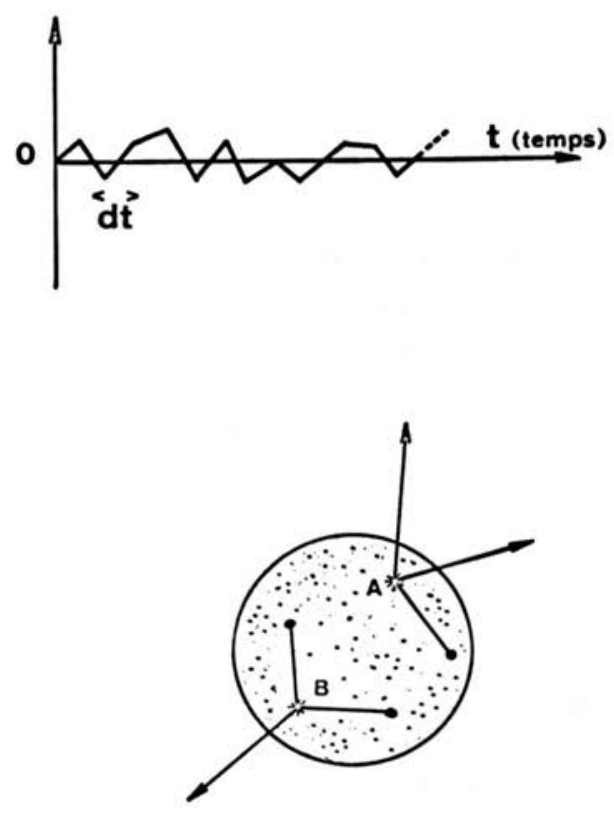

En A: plus de disparitions que d'apparitions

En B: plus dapparitions que de disparitions

FIG. I

Au catalogue des apparitions, il faut inscrire :

- les neutrons nouveaux engendrés par la fission,

- les neutrons " retardés », c'est-à-dire produits par activation hors fission,

- les neutrons réinjectés dans le milieu, soit par la présence de réflecteurs, soit par la proximité d'autres édifices fissiles.

Les premiers sont assez bien connus; on en connaît notamment la distribution statistique en nombre par fission ( $\nu$ ) (tableau 2) et plus communément la valeur moyenne $(\bar{v})$. L'une et l'autre variant selon le matériau fissile (235-U, $233-\mathrm{U}$, 239-Pu, etc...) [r].

Pour les seconds, quoique assez bien connus, de nombreuses mesures sont encore pratiquées [2] car leur rôle est capital (tableau 3); c'est grâce à eux que s'effectue le pilotage des réacteurs, entre autre celui des réacteurs rapides. Sans eux, la durée moyenne de vie des neutrons prompts est si brève (de l'ordre de $10^{-8}$ seconde) que leur prolifération exponentielle ne laisserait guère de latitude aux réactions du pilote, fût-il automatisé par l'électronique.

VOL. $8-\mathrm{N}^{\circ} 2$ 
TABLEAU 2

PourCENTAGES DE NEUTRONS PROMPTS ÉMIS DANS LA FISSION

\begin{tabular}{|c|c|c|}
\hline Nombre de neutrons émis & บ 235 & $\mathrm{Pu} 239$ \\
\hline 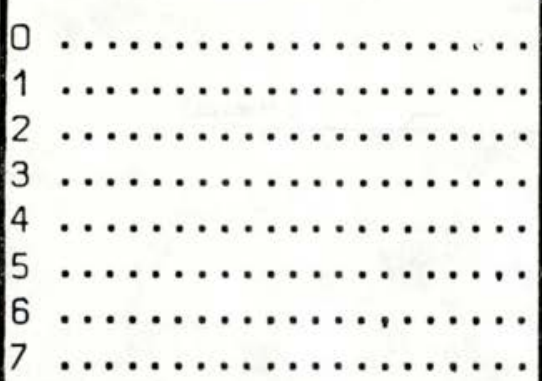 & $\begin{array}{r}2,7 \\
15,8 \\
33,9 \\
30,5 \\
13,3 \\
3,9 \\
0,1 \\
0,1\end{array}$ & $\begin{array}{r}0 \\
11 \\
13 \\
56 \\
11 \\
6 \\
5 \\
0\end{array}$ \\
\hline , p. 36 & & \\
\hline
\end{tabular}

TABLEAU 3

TAUX ET PÉRIODES DES NEUTRONS RETARDÉS

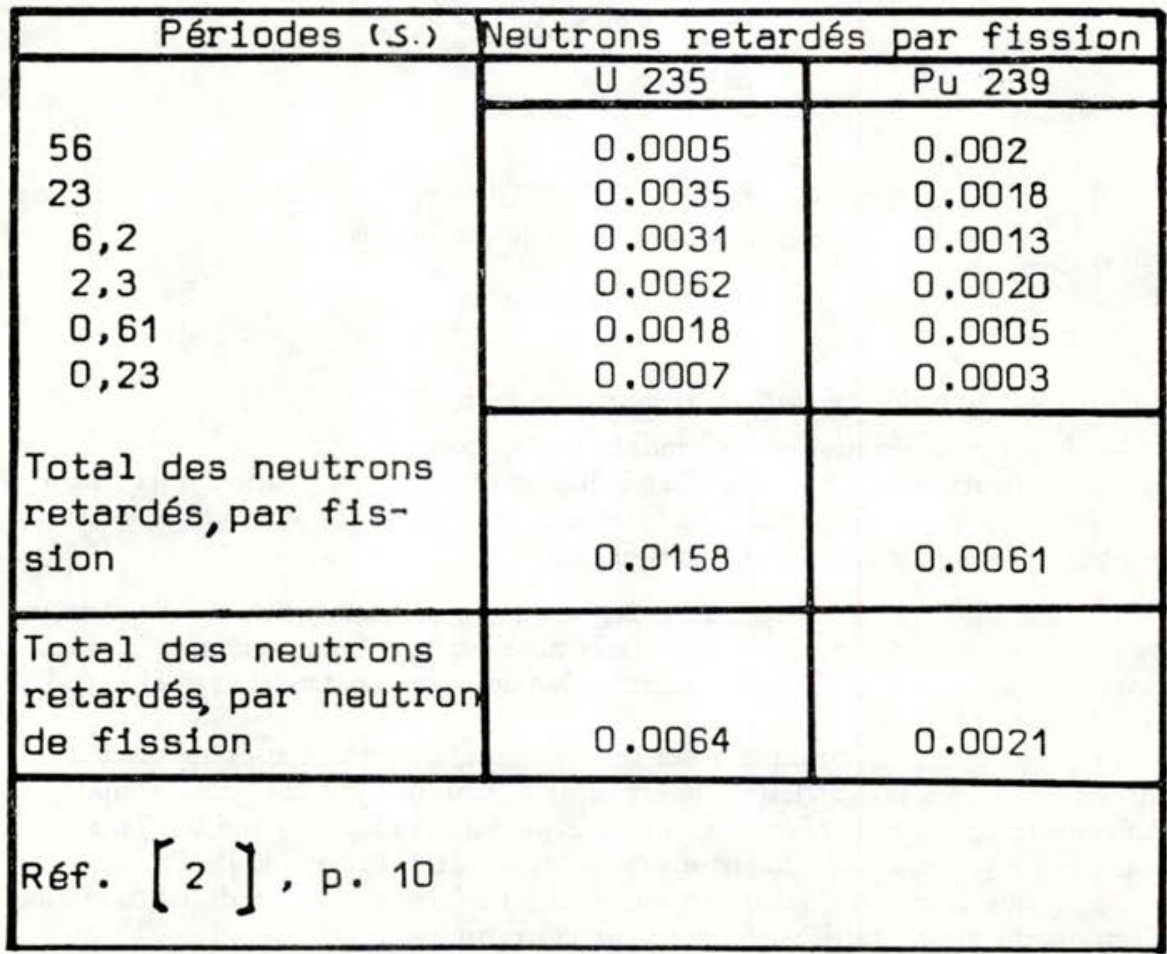


Il faudra donc distinguer par la suite, compte tenu des réinjections, l'état critique " prompt ", dû à la première catégorie de génération, de l'état critique " retardé"), dû à la fois à la première et à la seconde catégorie.

Au catalogue des disparitions, il faut inscrire :

- les absorptions neutroniques,

- les évasions de neutrons sans retour.

Les absorptions neutroniques sont la somme des captures et des fissions. Leur probabilité d'occurrence varie considérablement avec l'énergie des neutrons projectiles au moment de leur collision avec les noyaux cibles, ainsi qu'avec la nature de ces derniers : noyaux de matière fissile, du modérateur, du caloporteur, des matériaux de structure, etc. Les neutrons naissants, dotés d'une énergie élevée, perdent celle-ci à la suite de nombreuses collisions, élastiques et inélastiques, et la rapidité de cette dégradation est d'autant plus grande que la masse nucléaire des noyaux télescopés est plus faible. C'est ainsi que l'hydrogène est le modérateur le plus efficace (tableau 4), suivi du deutérium, du béryllium et du carbone pour ne citer que les plus couramment évoqués [3].

\section{Nombres moyens de collisions \\ (de $2 \mathrm{MeV}$ à $0,03 \mathrm{eV}$ ?}

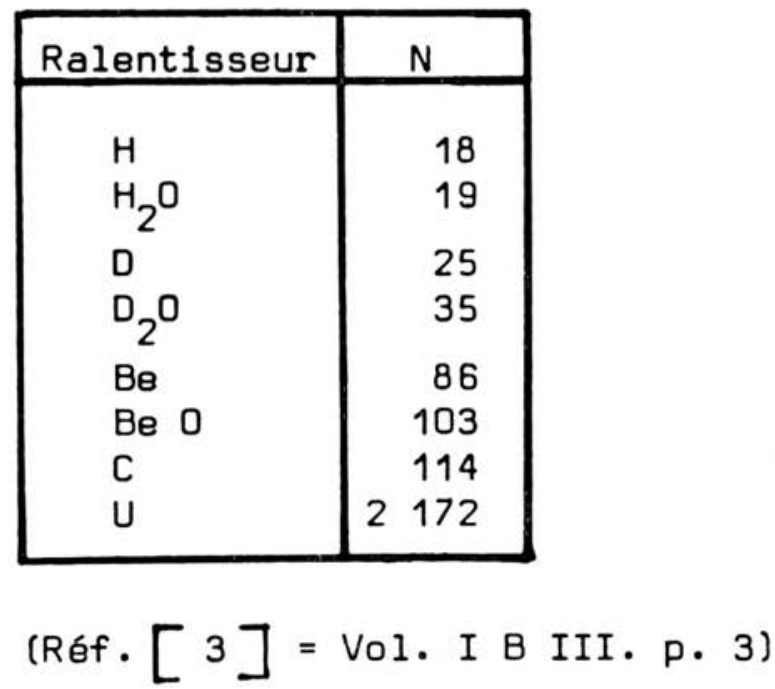

vOL. $8-\mathrm{N}^{0} 2$ 
Les évasions de neutrons dépendent essentiellement de la forme géométrique du milieu : à production constante, les fuites sont d'autant plus élevées que la surface d'évasion est grande et c'est ainsi qu'à volume constant une sphère laisse fuir moins de neutrons qu'un cylindre et que ce dernier en laisse moins fuir qu'un parallélépipède.

Le bénéfice de cet inventaire est de rappeler à quel point l'état critique dépend de nombreux paramètres : paramètres liés à la nature, à l'état et à la distribution de la matière, paramètres liés à la forme du milieu multiplicateur.

Souvent, en criticité, on rassemble les effets des premiers sous la forme condensée d'un coefficient de multiplication dit " infini » $(k \infty)$, car il ignore les limites matérielles du milieu multiplicateur; les effets des seconds sont rassemblés dans un terme correctif, $P$, dit anti-fuites; et la combinaison des deux constitue le coefficient de multiplication effectif $\left(k_{\mathrm{eff}}\right)$, qui, lorsqu'il est égal à l'unité, définit l'état critique [4].

$$
k_{\mathrm{eff}}=k_{\infty} \cdot P \quad(P \text { toujours }<\mathrm{I})
$$

\section{FACTEURS D'ÉVOLUTION}

La prévention de la surcriticité consiste essentiellement, comme le dirait M. de la Pallice, à faire en sorte qu'un milieu sous-critique ne devienne pas critique. Il y a donc lieu de considérer l'état initial des choses pour s'assures de sa sous-criticité, puis de suivre ses transformations intrinsèques (physiques, chimiques ou géométriques), ou extrinsèques, afin de vérifier que, fonctionnellement ou accidentellement, il n'évolue pas vers une forme s'approchant dangereusement de la criticité.

Les facteurs dominants sont l'enrichissement et la modération. L'enrichissement explicite la partie des produits fissiles qui subit la fission à toute énergie (et en fait presque entièrement à basse énergie), par opposition à celle qui ne la subit qu'à partir d'un certain seuil d'énergie généralement élevé : ainsi l'uranium 235 par rapport au 238. La modération représente la faculté qu'a le milieu de ralentir les neutrons, c'est-à-dire d'abaisser leur vitesse pour favoriser - comme au billard japonais - leur absorption, tantôt sous forme de capture, tantôt sous forme de fission. L'effet combiné de ces deux facteurs se lit aisément sur la courbe donnant le $k \infty$ de l'uranium (fig. 2), selon son enrichissement, en fonction de sa modération par l'eau (représenté ici par le rapport molaire $H / U$ ) [4]. On constate qu'il y a un lieu des maximums des $k \infty$, ce qui laisse présager qu'il y aura une masse critique minimale dans certaines configurations au moins. Si l'enrichissement ne peut guère subir d'évolution, on conçoit que la modération, elle, peut subir de fortes modifications et la concentration d'un produit fissile sera un des facteurs à surveiller le plus jalousement.

Les transformations physiques agissent sur le pouvoir multiplicateur du milieu, par exemple, lorsqu'il s'agit de métal, par le biais de la densité : les différences entre 


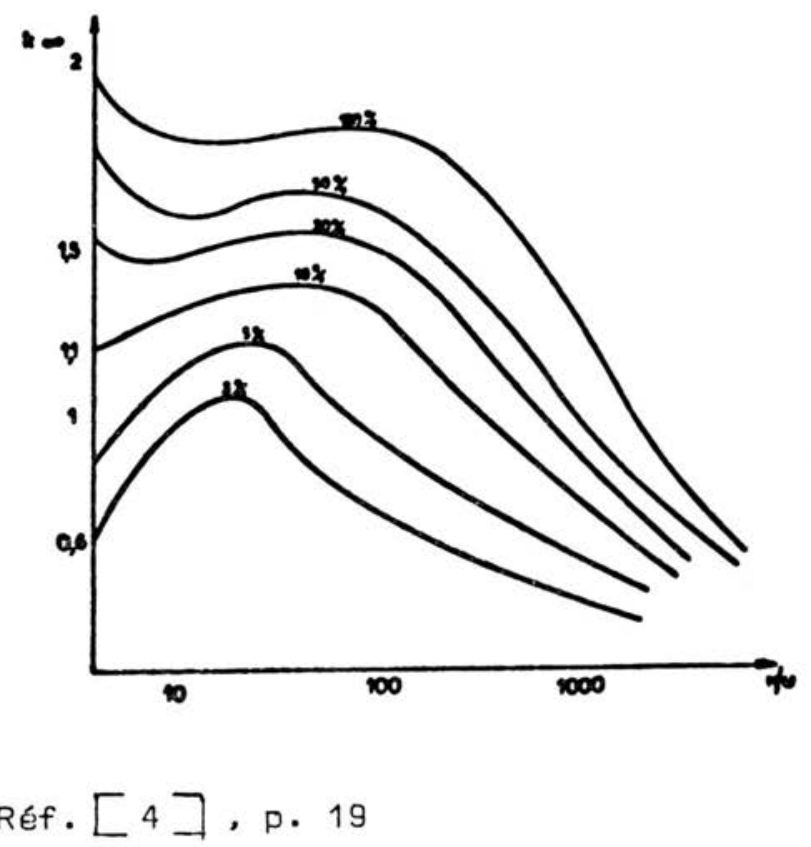

Fig. 2. - Influence de l'enrichissemert de l'uranium et de la modération par l'eau sur le $k_{\infty}$.

les variétés allotropiques $\alpha$ et $\delta$ du plutonium-239 (de densités respectives 19,6 et 15,7$)$ conduisent à des valeurs de masses sphériques critiques, totalement réfléchies par de l'eau, de 5,6 $\mathrm{kg}$ et 7,6 kg.

Les transformations cbimiques (par exemple de métal en fluorure, ou d'un degré d'oxydation à un autre), affectent à la fois les paramètres physiques (ainsi la densité) et nucléaires du milieu (par adjonction ou retrait de diffuseurs et d'absorbeurs); elles modifient souvent profondément son pouvoir multiplicateur.

Enfin les transformations géométriques se produisent lorsqu'il y a transfert de produit d'un récipient à un autre, rupture du récipient, dilatation, et leur effet sur le pouvoir multiplicateur est presque toujours déterminant.

A ces transformations en quelque sorte intrinsèques du milieu, il y a lieu d'ajouter les modifications extrinsèques telles que le rapprochement (ou l'éloignement) de réflecteurs nouveaux ou d'édifices fissiles distincts (interactions).

La liste exhaustive de tous les paramètres agissant sur la criticité serait longue, rébarbative, et en fin de compte illusoire car la plupart des facteurs réagissent les uns sur les autres. 


\section{MODES DE CONTRÔLE}

La prévention-criticité, faite à l'intention des exploitants d'installations nucléaires, ne pourrait s'accommoder d'une pareille complexité. Ayant affaire à de la matière fissile EN ÉVOLUTION, dans sa nature, sa forme et sa position (par opposition à ce qui se passe dans les réacteurs où elle est en quelque sorte figée dans sa nature et plutôt fixée dans sa position), la prévention criticité ne peut se concevoir qu'à l'intérieur d'une enveloppe qui limite les modifications tolérables des paramètres physiques, chimiques et géométriques accessibles aux opérateurs. C'est pourquoi elle énonce des RÈGLES FONDAMENTALES, qui concrétisent ces limites, à l'intérieur desquelles l'édifice sous-critique ne peut devenir, même accidentellement, critique; ou, à la grande rigueur, si on tolérait que par accident il devienne surcritique, cela n'entraînerait pas de conséquences dramatiques pour le personnel et le matériel.

Ces règles fondamentales s'appuient sur des principes, qui synthétisent le ou les modes de contrôle grâce auxquels la sûreté-criticité est assurée [r] [6] [7] :

- contrôle par la masse,

- contrôle par la concentration,

- contrôle par la géométrie,

- contrôle par l'empoisonnement.

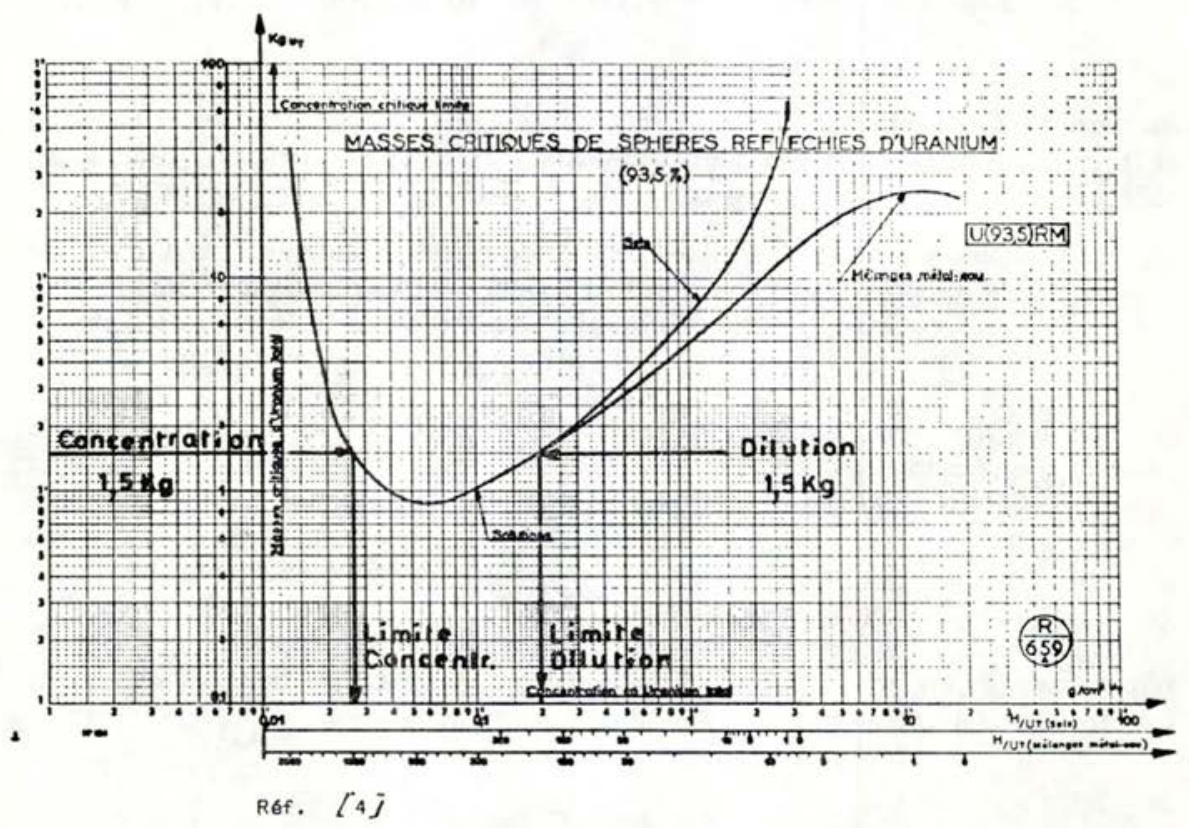

EIG. 3. - Limites de concentration. 
Des exemples simples illustreront cette méthode à l'aide des courbes établies ou rassemblées par le Service d'Etudes de Criticité.

La courbe de la figure 3 montre, pour des sphères d'uranium 235 à (93,5\%) la variation de la masse critique en fonction de la modération par de l'eau [4]. On voit clairement :

- qu'au-dessus d'une certaine concentration, il n'y a pas de divergence possible;

- que la fonction n'est pas monotone et qu'il y a un minimum de masse au-dessous duquel toute divergence est impossible.

Comme la sphère est 'de toutes les géométries celle qui favorise le moins les évasions, on peut être sûr qu'au-dessous de cette concentration, ou au-dessous de cette masse, le milieu multiplicateur ne peut certainement pas devenir surcritique. Encore faut-il s'assurer qu'on ne substituera pas au réflecteur hydrogéné de plus de $20 \mathrm{~cm}$ d'épaisseur un réflecteur plus efficace tel que le béryllium (fig. 4), ou l'eau lourde [4], ou le graphite nucléairement pur ce qui n'est pas - il faut en convenir - d'un usage très courant dans les usines ou ateliers traitant la matière fissile.

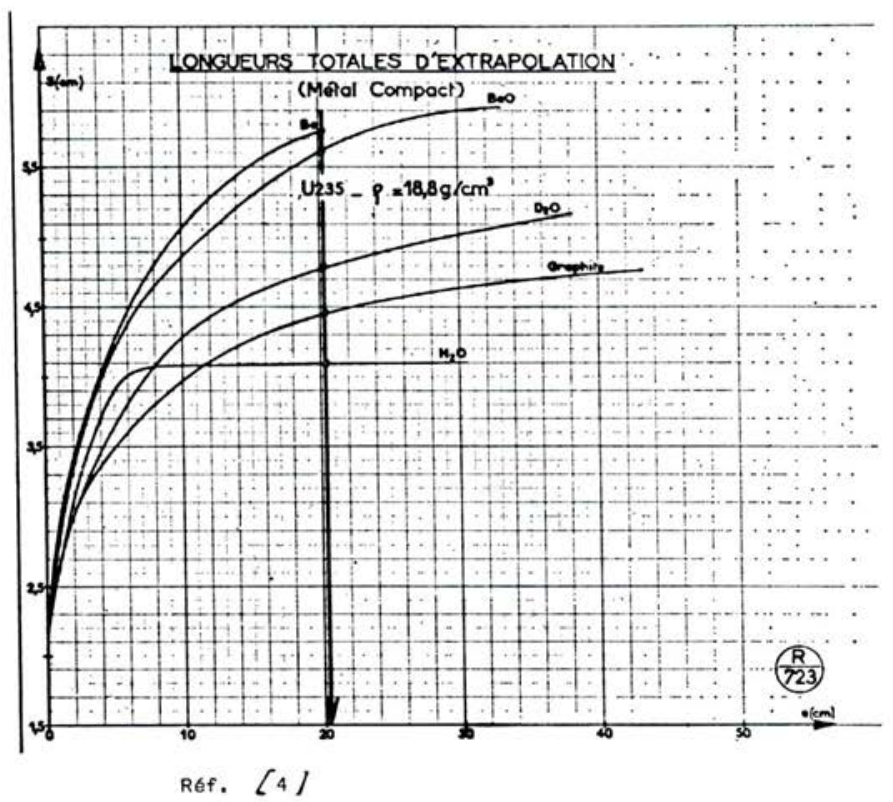

FIG. 4 - Pouvoir réflecteur (assimilé à la longueur d'extrapolation).

La courbe de la figure 3 montre également que si l'on travaille à masse constante, on peut, par dilution ou par évaporation, si on ne limite pas simultanément la concentration, aboutir à une divergence. La réaction instinctive est de se 
méfier de la concentration; mais - sauf si l'on est averti - on ne se méfiera pas de la dilution. Il faudra imposer dans ces deux derniers cas un double contrôle, celui de la masse et celui de la concentration (limite supérieure ou inférieure).

Les deux autres courbes montrent [8], à masse constante, l'effet de la géométrie pour des cylindres nus (fig. 5) ou enveloppés par 2,5 cm d'eau (fig. 6). On voit que du cylindre plat (type rondelle) au cylindre allongé (type barreau) le coefficient de multiplication effectif varie en passant par un maximum au voisinage de l'orthocylindre. Là encore, certaines masses seront sûres quelle que soit la forme; pour d'autres, il faudra, à forme géométrique donnée, limiter la masse.

Le mode de contrôle par l'empoisonnement consiste à introduire volontairement dans le milieu un puissant absorbeur tel que le bore (le plus souvent sous forme d'anneaux RASHIG en pyrex) destiné à abaisser la réactivité du milieu; il est alors essentiel de s'assurer de la permanence de cet absorbeur au cours du temps, et en particulier qu'il ne s'évade pas par lessivage.

Tous ces modes de contrôle aboutissent en fin de compte à démontrer, par des calculs éprouvés et vérifiés par des expériences, que le coefficient de multiplication effectif du milieu ( $k_{\text {eff }}$ ) est inférieur à un. La singularité de la prévention en sûreté-criticité réside dans cette notion de seuil : en dessous de un, si près qu'on s'en approche, l'édifice multiplicateur est sûr en matière de criticité; au-dessus, si peu qu'on s'en éloigne, on diverge et par conséquent on entre sans ambiguïté dans le domaine des accidents de criticité. C'est cette notion de seuil absolu qui distingue la sûreté-criticité de la sûreté radiologique par exemple, où, même si on fixe des valeurs limites telles que roo mrem par semaine ou 25 rems, le dépassement léger de ces limites, par exemple à i 20 millirems ou à 30 rems, n'entraîne pas un changement brutal d'ampleur dans les conséquences; ce qui n'est évidem-

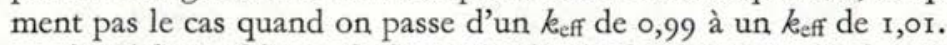

Aussi le problème de la certitude de l'estimation du $k_{\text {eff }}$ est-il primordial; celui-ci doit être évalué avec une fourchette d'erreur qui le place sans aucun doute possible, malgré la nature statistique des calculs effectués, au-dessous de l'unité.

A cette certitude mathématique, il faut ajouter la certitude " opérationnelle », c'est-à-dire la vérification du fait que les opérations décrites se dérouleront bien comme prévues, tant du point de vue technique, que du point de vue humain : c'est le contrôle des hypothèses. Si, avec de la minutie et du sens critique, on peut parvenir à la certitude technique que, par exemple, les dissolutions se font bien de façon homogène, que dans les solutions il n'y a ni floculation, ni sédimenration, etc.; par contre le facteur humain reste, par nature et quoi qu'on fasse, susceptible de défaillance.

Aussi, quand il ne peut être automatiquement et complètement éliminé - ce qui est fréquent - des coefficients de sécurité assurent cette garantie contre les défaillances prévisibles. Ainsi, dans un contrôle par la masse, pour pallier le risque du double chargement, utilise-t-on le facteur o,43 par rapport à la masse critique [4].

En conclusion, si les prévisions opérationnelles sont bien faites, les hypothèses bien vérifiées, les marges de précision des calculs connues avec certitude, les coefficients de sécurité éventuels convenablement appliqués, il NE PEUT survenir d'accident de criticité.

Et pourtant, il s'en est produit ! 


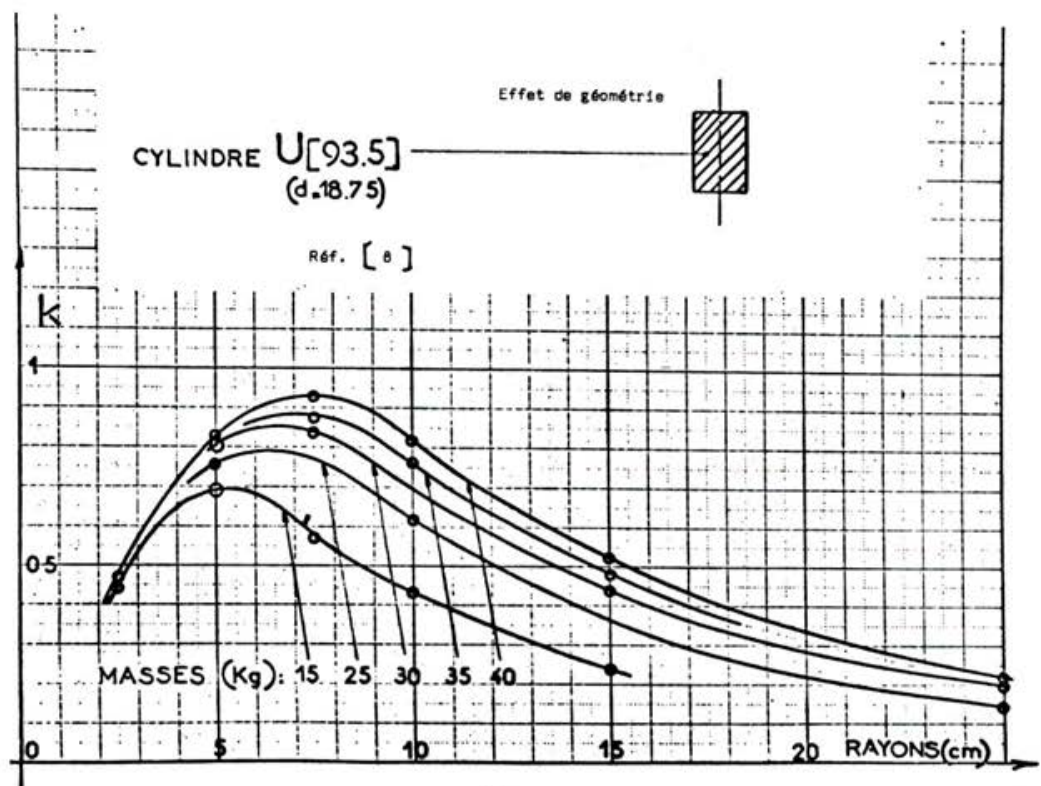

FIG. 5

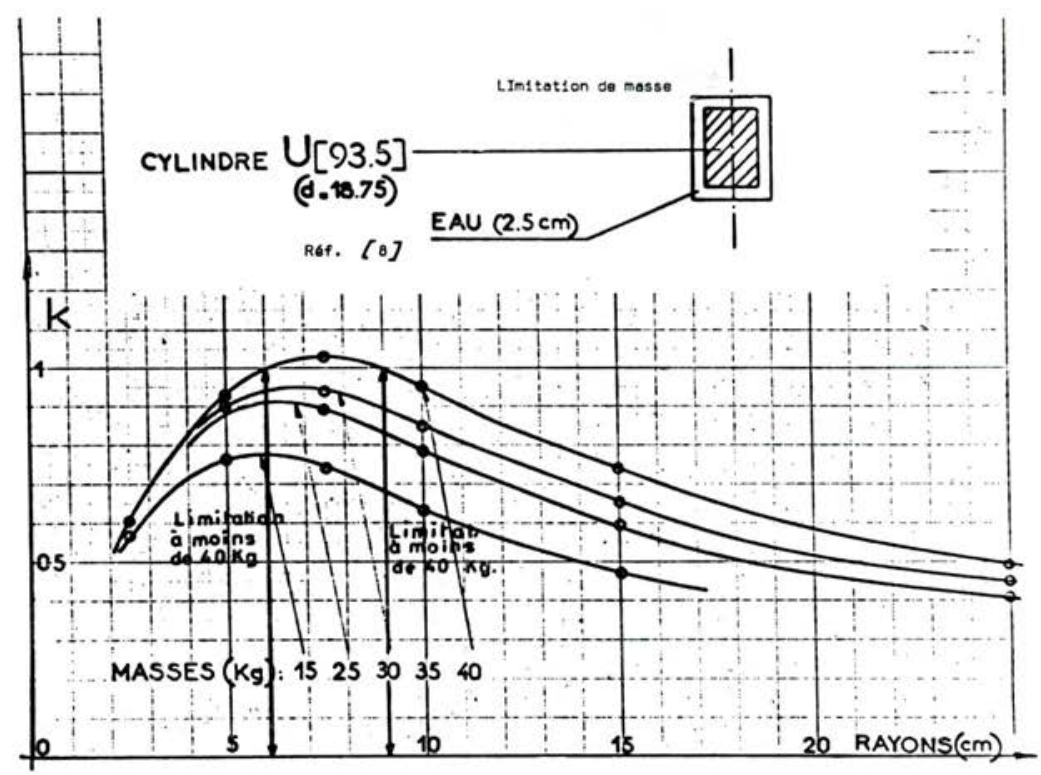

FIG. 6

vol. $8-\mathrm{N}^{\circ} 2$ 


\section{ACCIDENTS ET INCIDENTS}

C'est que, si fertile que soit l'imagination dans la prévision des incidents imaginables, si strictes que soient les consignes d'exploitation, l'imprévisible peut surgir de la faillibilité du cerveau et des muscles humains.

Deux exemples l'illustreront :

Le premier [9] est relatif à un accident survenu à Wood River Junction le 24 juillet 1954, dans une usine de récupération de combustible. Un technicien, par suite d'une lacune dans les consignes d'exploitation voulut verser, comme à l'accoutumée, onze litres de ce qu'il ignorait être ce jour-là de la solution concentrée de nitrate d'uranyle dans un réservoir non sûr contenant $4 \mathrm{I}$ litres de solution de carbonate de sodium.

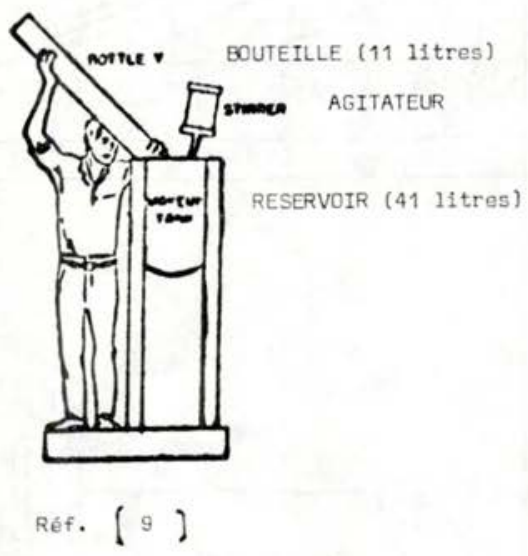

FIF. 7. - Accident de Wood River Junction (position du technicien).

En fait, une solution concentrée de nitrate d'uranyle avait été malencontreusement placée dans le même type de récipient que celui qui n'aurait dû servir qu'à des solutions faiblement contaminées; des consignes particulières prévoyaient qu'elle n'aurait pas dû être versée dans le réservoir incriminé. Le technicien, malgré l'étiquetage adéquat du flacon de ir litres, la prit cependant pour un résidu contaminé et non pour ce qu'elle était, c'est-à-dire une solution concentrée. L'excursion critique se produisit lorsque ro litres environ furent versés dans le réservoir de 4I litres où un agitateur de forte taille assurait l'homogénéité du mélange (fig. 7). Le technicien en mourut $49 \mathrm{~h}$ plus tard. A ce stade on notera :

- la vérification insuffisante des hypothèses techniques (cristallisation inhabituelle dans un évaporateur en amont dans la chaîne);

- la lacune des consignes générales (interdiction d'utiliser sans autorisation un appareillage non prévu, en l'occurrence le réservoir de $4 \mathrm{I}$ litres); 
- l'inattention vis-à-vis de la consigne particulière relative au réservoir (par suite du stockage de solutions concentrées au milieu des diluées).

Mais on ne relève ainsi que des erreurs décelables par analyse de la sûreté, et non une mise en défaut de l'imagination. Le bouquet vient après. En effet, quelques heures plus tard, un surveillant de l'installation qui, à deux reprises était entré dans le local une fois l'excursion terminée (un cinquième du volume de la solution avait été projeté hors du réservoir et le reste était par conséquent à un niveau sous-critique), ce surveillant donc y pénétra une troisième fois et, le plus naturellement du monde, arrêta l'agitateur qui, inutilement, continuait à tourner. Il déclencha ainsi - on l'imagine à sa stupéfaction ! - une deuxième excursion critique, celle-ci difficilement imaginable : en effet, en coupant le courant, il supprimait bulles et vortex qu'entretenait l'agitateur en marche. Que ce soit par changement de géométrie ou de densité, le milieu sous-critique était redevenu surcritique (fig. 8).

\section{$2^{\circ}$ phase de l'accident de criticité de Wood River Junction}

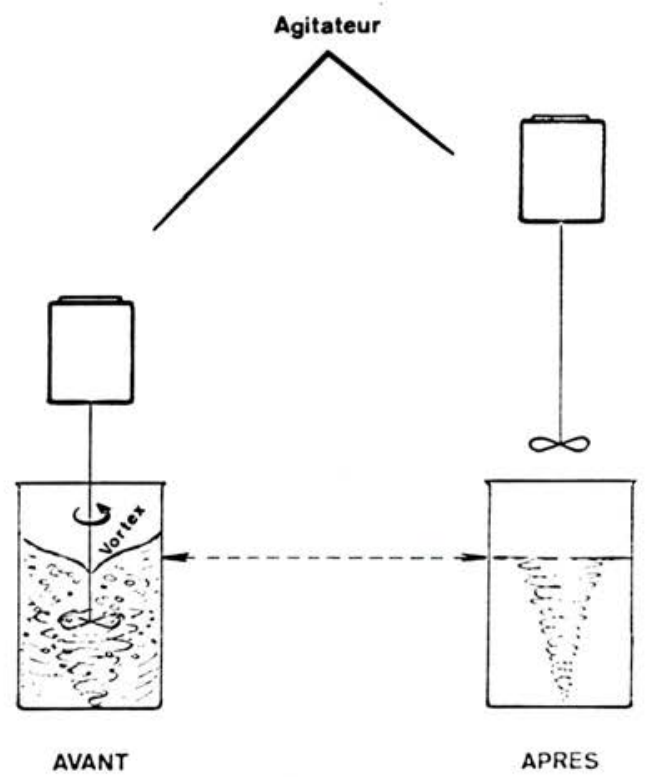

FIG. 8

Le deuxième exemple est relatif à un incident, qui - pour n'avoir pas eu de conclusion dramatique - n'en illustre pas moins la latence du risque de criticité. En juin 1965 , dans une installation du CEA dont j'avais alors à m'occuper [10], on procédait à un décapage chimique de pièces massives de plutonium. Après 
attaque par une solution très diluée d'acide chlorhydrique qui dissout bien ce métal, la pièce était plongée dans 25 littes d'acide nitrique concentré ro $\mathrm{N}$ qui le « passive ». Pour la pureté on avait choisi un produit Merck; et pour la concentration, deux contrôles quantitatifs, l'un par acidiméttie, l'autre par densimétrie, l'assuraient. Aucune dissolution notable ne pouvant alors se produire, la configuration plutonium-acide était sûre du point de vue criticité (fig. 9).

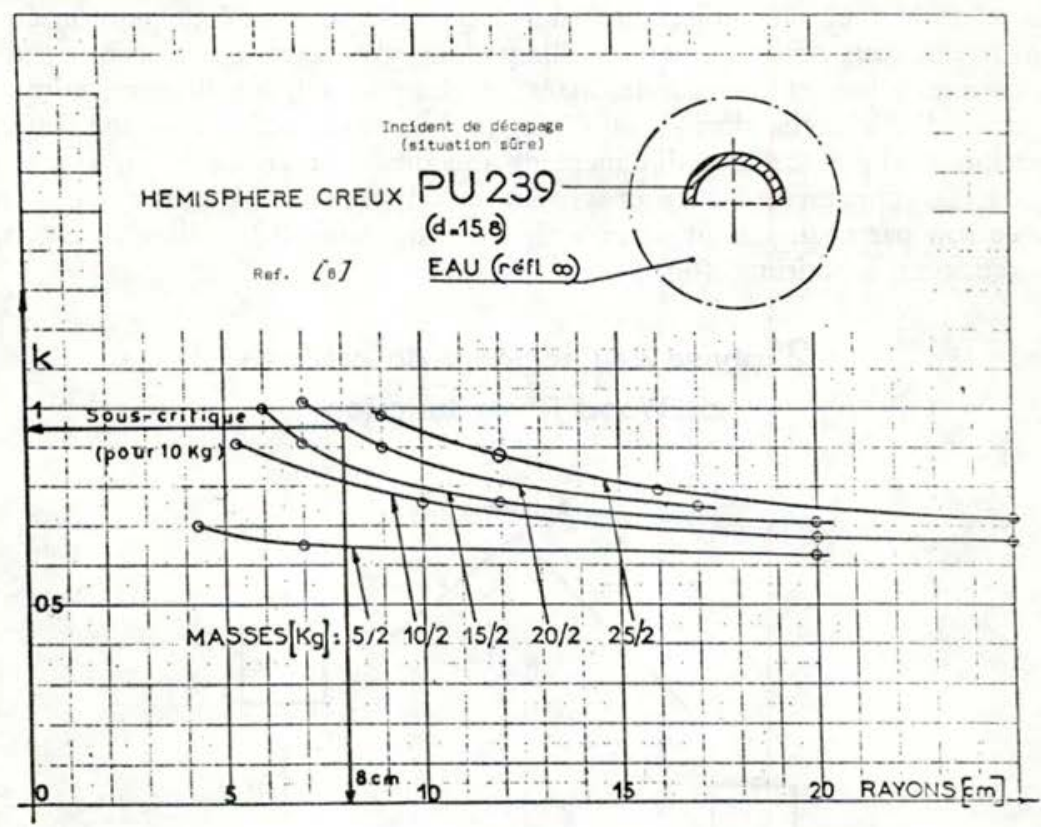

FIG. 9

Après de nombreuses opérations ainsi pratiquées sans incident avec renouvellement du bain nitrique, un jour, dès la plongée de la pièce métallique, d'abondantes et inattendues vapeurs suspectes se dégagèrent. L'opérateur vida aussitôt et instantanément le bac, soustrayant ainsi la pièce à l'agent corrodant imprévu, et évitant de ce fait l'excursion cutique qui se serait sans doute produite si la dissolution du plutonium s'était poursuivie plus avant. Que s'était-il passé? Après enquête on sut qu'un magasinier plein de zèle avait transféré l'acide nitrique Merck dans des bonbonnes apparemment vides ayant contenu de l'acide chlorhydrique, et dans lesquelles sans qu'il le sache, il en restait un fond; ensuite, naturellement, le tout fut dûment étiqueté. La présence d'acide chlorhydrique dans le nitrique du bain de passivation avait entraîné la dissolution rapide du plutonium, et, par bonheur, l'apparition du signal de danger constitué par l'émanation de vapeur.

A supposer qu'il y eut eu moins d'acide chlorhydrique, l'attaque serait passée inaperçue et l'accident eut été probable (fig. 10). Naturellement, à la suite de cet incident qui annihila les réticences antérieures des opérateurs, aux contrôles 
quantitatifs de la solution nitrique fut ajouté un contrôle qualitatif de tout nouveau bain avant plongée de matière fissile.

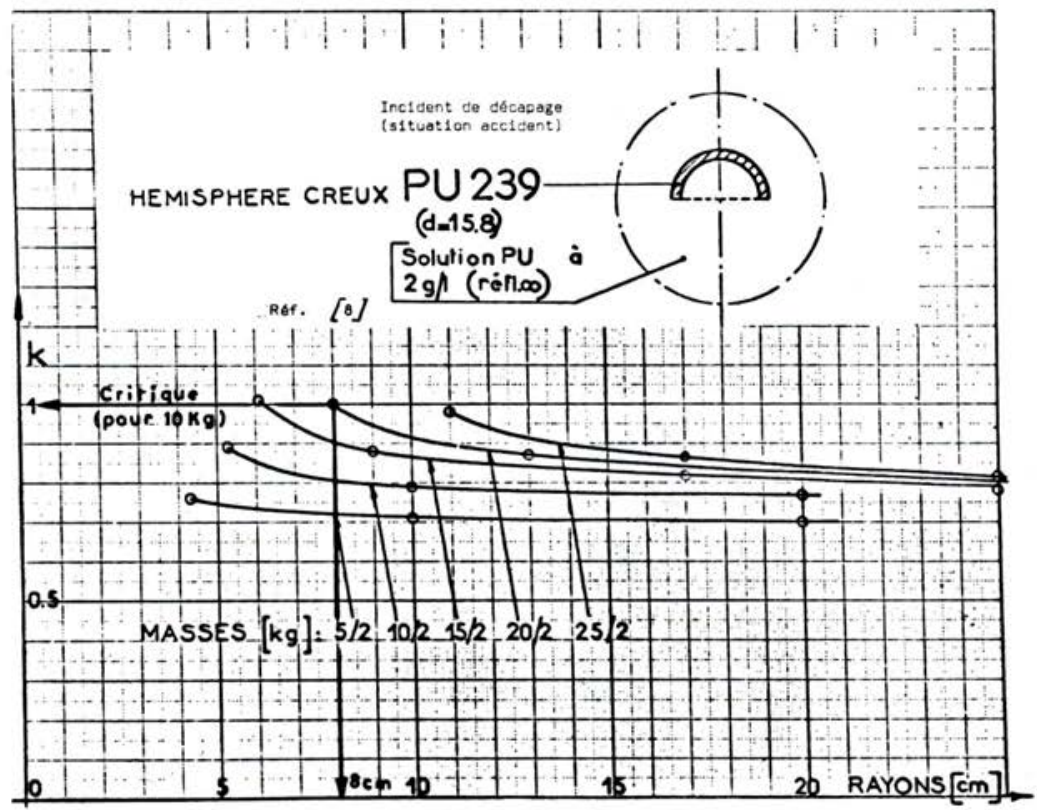

FIG. IO

Il faut retenir de ces incidents, que je crois instructifs, qu'il ne faut pas se fier aux apparences fussent-elles convaincantes, que la réalité dépasse parfois la fiction, et qu'une gêne vaut mieux qu'un accident.

\section{SYSTÈME D'ALARME-CRITICITÉ}

Puisqu'accident il peut y avoir, il faut dès lors bien s'en accommoder. Naturellement, il serait ridicule de ne prévoir que cela : des précautions nombreuses et sérieuses, des contrôles efficaces les évitent tant et si bien qu'en France, depuis l'origine, il ne s'est produit dans les laboratoires et usines AUCUN accident de criticité.

S'il devait s'en produire, nul ne saurait prédire quand et où - a fortiori pourquoi - ils auraient lieu. Mais vraisemblablement, ils seraient liés à l'erreur humaine, et lorsque les chances de mécomptes dans les manipulations augmentent, il est parfois prudent de prévoir un système d'alarme.

Et tout d'abord, quand et à qui donner l'alerte ? A la question quand, la réponse est : dans des délais compatibles avec la durée des réflexes humains, évalués à environ une demi-seconde. A qui ? bien entendu en priorité aux opérateurs les plus proches de la source, le plus proche éventuel se trouvant à environ 
$50 \mathrm{~cm}$ de l'édifice surcritique si, par exemple, il manipule en boîte à gants. Dès lors on se trouve devant un dilemme : ou bien l'excursion critique se développe avec un temps de doublement de puissance tellement court qu'il n'y a aucune chance d'y soustraire l'opérateur le plus proche, et dans ce cas l'alerte ne vaut que pour les suivants; ou, au contraire, il est encore possible pour lui de réagir. Partant de la dose létale de 400 rads pour 50\% des irradiés par les neutrons et les gammas, on en déduit que si le développement de l'excursion n'est pas trop rapide, et qu'il y a une demi-seconde pour réagir et une autre pour s'éloigner, il faut prévenir la victime potentielle la plus exposée au pire au niveau de 100 rads, soit 25 rads à I mètre. D'autre part, dans l'ignorance où l'on est du point précis où se produira l'accident, il faut que la " portée » du détecteur le plus proche soit capable de le révéler. Pour ne pas en multiplier incongrûment le nombre, compte tenu de la surface des laboratoires et usines, on a estimé que 30 mètres d'espacement, si les détecteurs sont à vue, est un critère raisonnable. Dès lors la sensibilité de n'importe quel détecteur doit être d'au moins 25 mrad. En fait, il y a toujours plus ou moins d'obstacles entre le détecteur et la source d'accident. De plus, pour éviter les fausses alarmes génératrices de risques inutiles (et c'est en cela que réside la difficulté majeure de la détection des accidents de criticité), il faut qu'au moins deux détecteurs soient sensibilisés pour que l'alerte ne soit pas déclenchée de façon intempestive; ce qui ramène la distance récurrente entre détecteurs d'une même chaîne à plutôt io ou is mètres qu'à 30 .

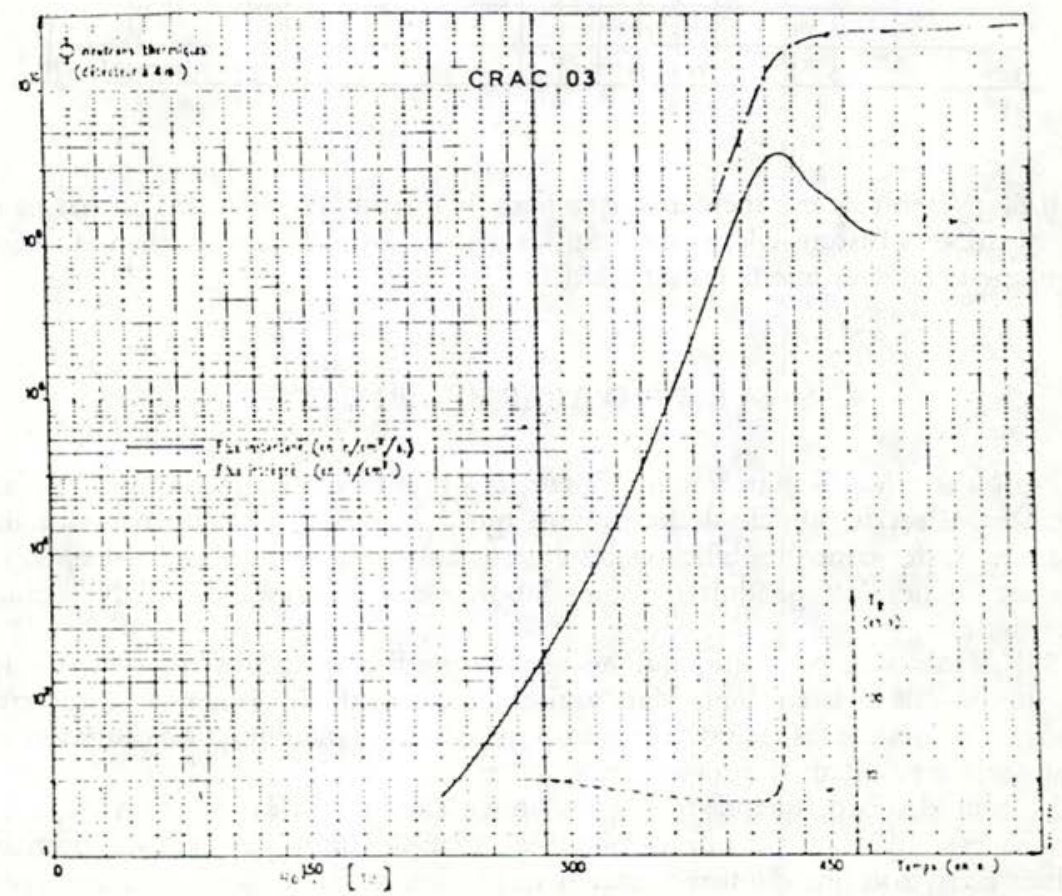

FIG. II. - Dynamique d'excursion critique. 
Dans le passé, sous l'impulsion de M. LAvIE, une première génération de détecteurs d'alerte avait été développée, sur des critères d'ailleurs différents, et rend encore d'appréciés services [II] : mais elle reposait avant tout sur le postulat d'un développement rapide de l'excursion critique [1 2]. Aussi, les détecteurs répondaient-ils pourvu que deux d'entre eux soient sensibilisés dans un délai de coïncidence d'une demi-seconde. Ce principe a été mis en défaut à la suite des expériences CRAC effectuées par le Service d'Etudes de Criticité [1 3 ], expériences qui mettaient en évidence des temps de montée beaucoup plus longs (fig. II).

\section{Déclenchement des sondes de criticité \\ (Influence du temps de doublement et du réglage des seuils des détecteurs )}

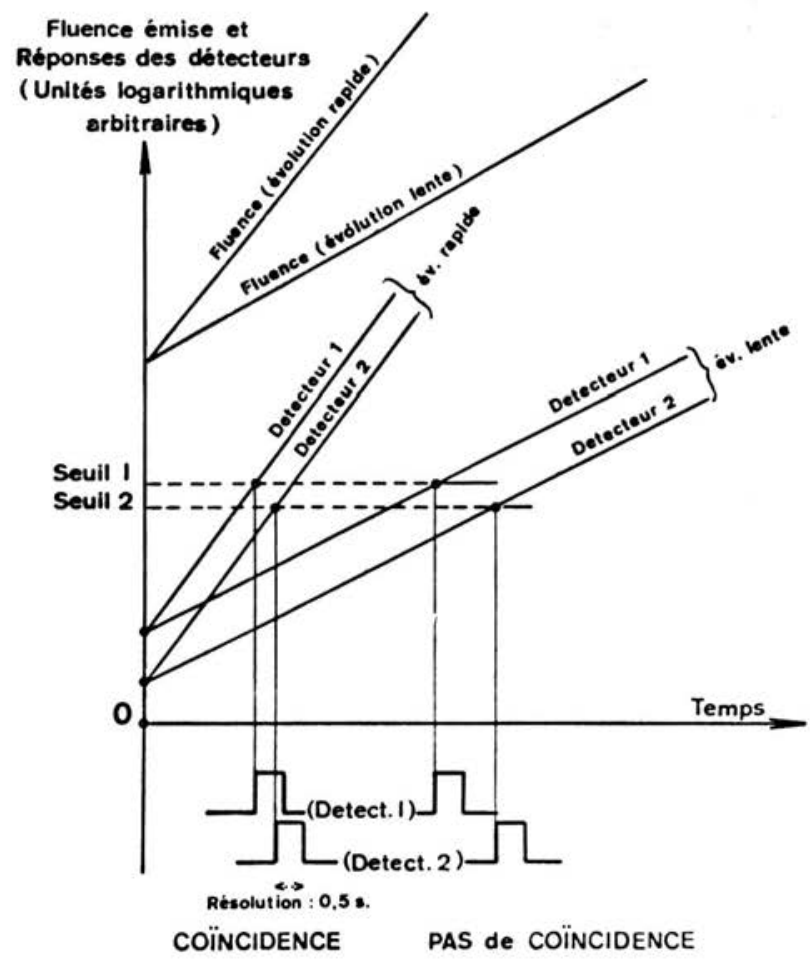

FIG. 12

Cette dynamique, en raison des écarts techniques de réglage des seuils des détecteurs, rend inopérant le processus de coïncidence avec une résolution aussi courte (fig. 12). Il a donc fallu, dans un premier temps pallier ce défaut en allongeant la durée de résolution des coïncidences révélatrices. 
Dans un deuxième temps, compte tenu de désuétude prochaine des détecteurs en service, MM. RENARD et PRIGENT [14] ont étudié et sont parvenus à mettre au point une deuxième génération de détecteurs qui respecte les spécifications techniques essentielles de la première génération (réponse en dose, déclenchement par deux sur $N$ informations), mais qui :

- la modifie, en ce sens qu'à la coïncidence de deux informations est substituée leur concordance (ou leur superposition) [ $\mathrm{r}$ s], la seconde authentifiant la première quel que soit l'intervalle de temps les séparant; dès lors, la précision du réglage des seuils de déclenchement des sondes n'a plus à être aussi sévère qu'auparavant, et on s'affranchit de la nécessité de corréler le temps de doublement et le délai de coïncidence (résolution);

- l'améliore techniquement (réponse additive en dose neutrons plus gammas, robustesse, légèreté, etc.); mais il n'en restera pas moins qu'on ne peut impunément abaisser le réglage du seuil de détection en dose, sous peine de mécomptes pour des accidents à évolution lente (fig. 13);

- sera sans doute moins chère (du moins en ce qui concerne l'ensemble électronique qui délivre l'information à partir de laquelle seront actionnés les signaux d'alerte).

\section{Influence du temps de doublement $(\tau)$ \\ sur le seuil de déclenchement \\ des sondes de criticité}

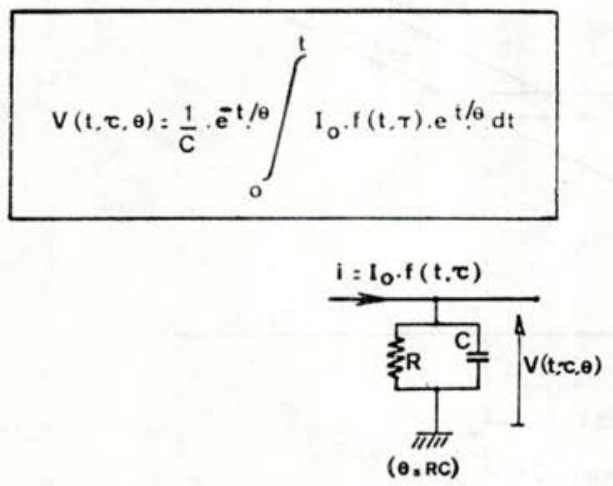

FIG. 13

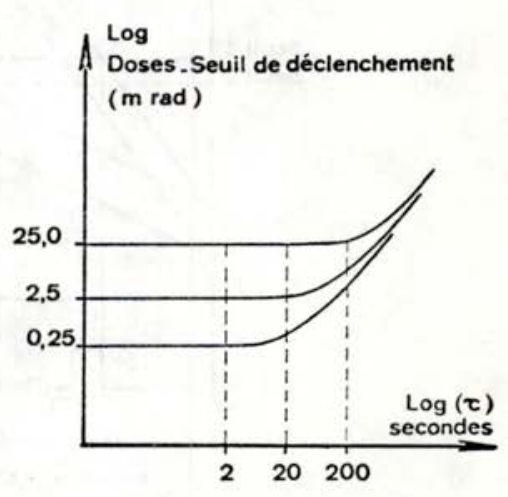

Ainsi espère-t-on soustraire à l'accident à développement rapide, sinon les malchanceux qui se trouveraient à proximité immédiate et pour lesquels aucun dispositif n'est actuellement concevable, du moins ceux qui, étant à plus d'un mètre, ne recevraient au pis que 25 rads. Que faire alors de ceux-ci, qui malgré tout, ont subi une irradiation significative? 


\section{DOSIMÉTRIE DE L'ACCIDENT CRITIQUE}

Nous entrons alors dans le domaine des réparations sur le matériel humain. Une donnée capitale pour la thérapeutique à appliquer est la connaissance des doses intégrées en divers points de l'organisme selon la nature, l'intensité et le spectre d'énergie du rayonnement : je pense que M. DoussET, qui nous a clairement et brillamment montré dans une récente réunion scientifique l'importance de ces paramètres, ne me démentira pas.

La reconstitution de l'accident n'est pas toujours possible, ni même parfois souhaitable; tout au moins peut-on tenter d'en reconstituer les effets. La variété des dosimètres gammas répond assez bien à une partie de la question, pourvu qu'on sache éliminer les effets de l'activation neutronique; mais pour les neutrons, la réponse est moins facile. La présence du corps humain (excellent modérateur et réflecteur de neutrons : $60 \%$ en poids d'eau) [16], les parois des bâtiments, et bien d'autres causes modifient le spectre incident et le spectre absorbé, tant des individus que des détecteurs, y compris ce détecteur particulièrement qualifié qu'est le corps humain par le sodium de son sang et le soufre de ses phanères. Il existe au niveau des individus, des dosimètres individuels bien connus (dits PS-I-C) développés par le STEPPA, et, dans les bâtiments des dosimètres de zone non moins connus dits SNAC, mis au point par M. BRICKA [17]; les uns et les autres sont constitués de détecteurs à activation (cuivre, or, indium) dont certains à seuil (soufre en particulier), blindés ou non par du cadmium, qui permettent de reconstituer avec une assez bonne précision le spectre neutronique absorbé et, par là, de fournir des éléments au calcul de l'exposition. Des ceintures de criticité de divers modèles, mais le plus souvent constituées avec du soufre, permettent d'espérer la reconstitution au moins sommaire de la position angulaire de l'individu par rapport à la source. Mais tout est ensuite affaire d'interprétation de ces mesures physiques, corrélées aux mesures de caractère biologique que perfectionne Mme Parmentrer. Dans ce domaine, le but ultime, qui est la connaissance exacte et localisée des doses absorbées par l'accidenté, en vue du pronostic et de la thérapeutique consécutive, n'est pas encore toute à fait atteint. Ce sera le fruit du travail du Groupe SILÈNE, qui opèrera dans un proche avenir autour de l'ensemble critique ainsi baptisé, fonctionnant en régime pulsé ou continu, et mis en place à VALDUC par le Service d'Etudes de Criticité.

\section{CONCLUSIONS}

L'exposé que je termine n'a pas eu - vous vous en doutez - l'ambition insensée d'être exhaustif; de nombreux et importants aspects des problèmes de criticité n'y figurent pas; certains ont même été délibérément négligés car généralement bien connus des radioprotectionnistes : c'est le cas de la dosimétrie. Par

VOL. $8-\mathrm{N}^{\circ}{ }_{2}$ 
contre j'ai tenté d'illustrer dans ce qui précède des idées qui me paraissent essentielles :

- la première, c'est qu'il faut proscrire du vocabulaire le couple de mots (lorsqu'il prétend à l'universalité) de « masse critique »; il n'y a en effet de critique que des configurations déterminées par leurs éléments physiques, chimiques et géométriques, dans un environnement défini; les masses critiques n’en sont que les conséquences;

- la deuxième, c'est qu'une configuration initialement sous-critique peut évoluer, de façon concertée ou non, vers un état plus ou moins proche de l'état critique, sous l'effet des modifications physiques, chimiques ou géométriques, ou encore par modification de son environnement primitif; il ne suffit donc pas d'examiner " statiquement » un édifice fissile;

- la troisième, c'est qu'il faut de l'imagination, toutefois tempérée par la logique, pour faire la "chasse» aux modifications possibles d'un édifice souscritique en vue de le soustraire en toutes circonstances à la promiscuité dangereuse de l'état critique : souvent le vraisemblable peut être contredit par l'inattendu;

- la quatrième, c'est que s'il se produit un accident, on ne saura par avance ni où ni quand (et on ne saura comment qu'après); mais il faut croire que ce défi permanent a été bien relevé en France puisqu'il ne s'y est encore produit aucun accident critique dans les usines ou laboratoires;

- la cinquième, c'est qu'il ne faut pas demander à l'alarme-criticité de faire plus qu'elle ne le peut : une excursion se développant avec la brièveté de l'éclair ne laisserait aucune latitude de fuite au personnel malencontreusement situé; par contre, toutes les autres personnes pourraient être soustraites à temps à l'irradiation irréversible, et même les plus exposées, si l'excursion se développe suffisamment lentement.

Pour conclure : la mise en œuvre de la sûreté-criticité, qui a fait depuis des années la démonstration de son efficacité, n'exige de ceux qui la pratiquent ni complaisance aveugle ni défiance paralysante; l'équilibre mental est ici encore le plus sûr garant de la santé des corps.

(1 2 juin 1973)

\section{BIBLIOGRAPHIE}

[1] Salmon J. et al. Théorie cinétique des neutrons rapides. Bibliothèque des Sciences et Techniques Nucléaires. INSTN, Presses Universitaires de France, éditeur.

[2] Reactor Handbook (vol. III, Part. A Physics), par SoodaK H. Interscience publishers (1962).

[3] Cours de Génie Atomique (vol. r) INSTN. Presses Universitaires de France, éditeur.

[4] Guide de Criticité. Rapport français CEA-R-3114.

[5] La Criticité. Plaquette éditée par le SPRAR, Marcoule (chapitre 3, p. 17).

[6] GEY A. et al. Sûreté Criticité : Recommandations concernant les fours de traitement de matière fissile. Note française CEA-N-1260.

[7] LeCORCHÉ P. et al. Sûreté Criticité : Recommandations concernant les stockages de matière fissile. Note française CEA-N-r 29r.

[8] Gey A., Moreau J., Parisot B. (Note DPSR) : coefficient de multiplication neutronique de quelques configurations usuelles. 
[9] Auxier J.A. Nuclear Accident at Wood River Junction. Nuclear Safety, vol. 6, $\mathrm{n}^{\circ}{ }_{3}$ (1965).

[10] GEY A. Communication privée. Compte rendu d'incident ( 1965$)$.

[ir] Bulletin d'Informations Scientifiques et Techniques du CEA, $\mathrm{n}^{\circ} 96$.

[12] LAvie J.M. et al. Détection des accidents de criticité. Note SCRGR/PAS de septembre 1962.

[13] Collection des résultats d'expériences CRAC (Notes SEEC).

[i4] Renard C., Prigent R. et al. Système de détection et d'alarme des excursions nucléaires accidentelles. Communication IAEA/SM-I43. Colloque de Vienne, 23-27 novembre (1970).

[15] Compte rendu de la $83^{\mathrm{e}}$ réunion de la Sous-Commission des Masses Critiques. Point IV.

[16] Recommandations de la Commission Internationale de Protection Radiologique. Rapport du Comité II, p. I58, Gauthier-Villars éditeur (1959).

[17] BRICKA M., CERCY J. : Le spectromètre neutrons à activation (SNAC) et sa réponse sur modérateur. Communication IAEA/SM-58.40. Colloque de Vienne (1965). 